\title{
Podolsky's electromagnetic theory on the null-plane gauge
}

\author{
M. C. Bertin*, B.M. Pimentel* and G. E. R. Zambrano ${ }^{\dagger}$ \\ *Instituto de Física Teórica - São Paulo State University \\ Caixa Postal 70532-2, 01156-970, São Paulo, SP, Brazil \\ ${ }^{\dagger}$ Departamento de Física, Universidad de Nariño \\ Calle 18 Cra 50, San Juan de Pasto, Nariño, Colombia
}

\begin{abstract}
Following the Dirac's technique for constrained systems we performed a detailed analysis of the constraint structure of Podolsky's electromagnetic theory on the null-plane coordinates. The null plane gauge condition was extended to second order theories and appropriate boundary conditions were imposed to guarantee the uniqueness of the inverse of the constraints matrix of the system. Finally, we determined the generalized Dirac brackets of the independent dynamical variables.
\end{abstract}

Keywords: Podolsky's electromagnetic theory, Null-Plane Gauge Conditions

PACS: <11.15.-q, 03.50.De, 41.20.-q>

\section{INTRODUCTION}

The Podolsky's electromagnetic theory [1] was developed as a generalization of the Maxwell's electromagnetism. It adds to the Maxwell Lagrangian a quadratic term of the divergence of the field-strength tensor in order to avoid divergences such as the electron self-energy and the vacuum polarization current. The resulting theory is the only possible generalization of the electromagnetic field that preserves invariance under $U(1)$ [2]. The Lagrangian density for Podolsky's theory is

$$
\mathscr{L}=-\frac{1}{4} F_{\mu \nu} F^{\mu \nu}-a^{2} \partial_{\lambda} F^{\mu \lambda} \partial^{\gamma} F_{\mu \gamma}, \quad \quad F_{\mu \nu} \equiv \partial_{\mu} A_{v}-\partial_{v} A_{\mu} .
$$

The theory yields field equations that are still linear in the fields, and predicts the existence of massive photons proportional to the inverse of the Podolsky's parameter $a$. The first consistent approach to the quantization of the field was made in instant-form by Galvão and Pimentel [3], meanwhile the authors studied the theory on the null-plane coordinates where Dirac's canonical formalism [4] was used to derived the correct gauge choice for this theory [5].

\section{CANONICAL STRUCTURE}

From the Lagrangian density (1), it is possible to calculate the canonical momenta

$$
\begin{gathered}
p^{\mu}=F^{\mu+}+2 a^{2}\left(\eta^{\mu-} \partial_{-} \partial_{\lambda} F^{+\lambda}+\eta^{\mu i} \partial_{i} \partial_{\lambda} F^{+\lambda}-2 \partial_{-} \partial_{\lambda} F^{\mu \lambda}\right) \\
\text { CP1296, XI Hadron Physics } \\
\text { edited by M. Nielsen, F. S. Navarra, and M. E. Bracco } \\
\text { (c) } 2010 \text { American Institute of Physics 978-0-7354-0848-7/10/\$30.00 }
\end{gathered}
$$




$$
\pi^{\mu}=-2 a^{2} \eta^{\mu+} \partial_{\lambda} F^{+\lambda}
$$

associated to the fields $A_{\mu}$ and $\bar{A}_{\mu} \equiv \partial_{+} A_{\mu}$ respectively. From this relations we are able to define the primary constraints of the theory

$$
\begin{aligned}
& \phi_{1}=\pi^{+} \approx 0 \\
& \phi_{2}^{i}=\pi^{i} \approx 0 \\
& \phi_{3}=p^{+}-\partial_{-} \pi^{-} \approx 0, \\
& \phi_{4}^{i}=p^{i}-\partial_{i} \pi^{-}+F_{--}-4 a^{2} \partial_{-}\left[\partial_{i} \bar{A}_{-}-2 \partial_{-} \bar{A}_{i}+\partial_{i} \partial_{-} A_{+}-\partial_{j} F_{i j}\right] \approx 0 .
\end{aligned}
$$

The canonical Hamiltonian density can be expressed by

$$
\begin{aligned}
\mathscr{H}_{c}= & p^{\mu} \bar{A}_{\mu}+\pi^{-}\left(\partial_{-} \bar{A}_{+}-\partial^{i} \bar{A}_{i}+\partial^{i} \partial_{i} A_{+}\right)-\frac{1}{2}\left(\bar{A}_{-}-\partial_{-} A_{+}\right)^{2}-\left(\bar{A}_{i}-\partial_{i} A_{+}\right) F_{-i} \\
& +\frac{1}{4} F_{i j} F^{i j}-a^{2}\left(\partial_{i} \bar{A}_{-}-2 \partial_{-} \bar{A}_{i}+\partial_{i} \partial_{-} A_{+}-\partial_{j} F_{i j}\right)^{2} .
\end{aligned}
$$

With the canonical Hamiltonian $H_{c}=\int d^{3} x \mathscr{H}_{c}(x)$ and the primary constraints (3) we build the primary Hamiltonian

$$
H_{P} \equiv H_{c}+\int d^{3} x u^{a}(x) \phi_{a}(x) \quad . \quad\{a\}=\{1,2,3,4\} .
$$

The consistency conditions on the constraints determine that the condition $\dot{\phi}_{1} \approx 0$ gives just the constraint $\phi_{3} \approx 0$, which is already satisfied. The consistency for the remaining constraints give equations for some Lagrange multipliers and a secondary constraints appears: $\chi \equiv \partial_{-} p^{-}+\partial_{i} p^{i} \approx 0$. From this relation no more constraints can be found. The analysis leave us with the following set of constraints:

$$
\begin{aligned}
\chi & =\partial_{-} p^{-}+\partial_{i} p^{i} \approx 0, \\
\phi_{1} & =\pi^{+} \approx 0 \\
\phi_{2}^{i} & =\pi^{i} \approx 0 \\
\phi_{3} & =p^{+}-\partial_{-} \pi^{-} \approx 0, \\
\phi_{4}^{i} & =p^{i}-\partial_{i} \pi^{-}+F_{i-}-4 a^{2} \partial_{-}\left[\partial_{i} \bar{A}_{-}-2 \partial_{-} \bar{A}_{i}+\partial_{i} \partial_{-} A_{+}-\partial_{j} F_{i j}\right] \approx 0 .
\end{aligned}
$$

It happens that $\chi$ and $\phi_{1}$ are first-class constraints, while $\phi_{2}^{i}, \phi_{3}$ and $\phi_{4}^{i}$ are secondclass ones. However, constructing the matrix of the second-class constraints we found that it is singular of rank four, which indicates that there must be a first-class constraint, associated with the zero mode of this matrix, and its construction is made from the corresponding eigenvector which gives a linear combination of second-class constraints. The combination happens to be just $\Sigma_{2} \equiv \phi_{3}-\partial_{i} \phi_{2}^{i}$ and it is independent of $\chi$ and $\phi_{1}$. Therefore, we have the renamed set of first-class constraints

$$
\begin{aligned}
& \Sigma_{1} \equiv \pi^{+} \approx 0, \\
& \Sigma_{2} \equiv p^{+}-\partial_{-} \pi^{-}-\partial_{k} \pi^{k} \approx 0, \\
& \Sigma_{3} \equiv \partial_{-} p^{-}+\partial_{i} p^{i} \approx 0,
\end{aligned}
$$


and a set of irreducible second-class constraints

$$
\begin{aligned}
& \Phi_{1}^{i} \equiv \pi^{i} \approx 0, \\
& \Phi_{2}^{i} \equiv p^{i}-\partial_{i} \pi^{-}+F_{i-}-4 a^{2} \partial_{-}\left[\partial_{i} \bar{A}_{-}-2 \partial_{-} \bar{A}_{i}+\partial_{i} \partial_{-} A_{+}-\partial_{j} F_{i j}\right] \approx 0 .
\end{aligned}
$$

The second-class constraints do not appear in the instant-form dynamics for this theory, thus, they are a common effect of the null-plane dynamics [7].

\section{NULL-PLANE GAUGE FIXING AND DIRAC'S BRACKETS}

Now let us eliminate the arbitrary functions from our theory by using gauge invariance to fix the remaining three degrees of freedom corresponding to the first class constraints (7). Let us choose the null-plane gauge defined by [8]

$$
A_{-} \approx 0
$$

The time preservation of this condition is equivalent to setting

$$
\partial_{+} A_{-}=\bar{A}_{-} \approx 0
$$

whereas the consistency requires

$$
\partial_{+} \bar{A}_{-}=\partial_{-}^{x} \bar{A}_{+}+\partial_{l}^{x} \bar{A}_{l}-\partial_{l}^{x} \partial_{l}^{x} A_{+} \approx 0 .
$$

The relations $(9,10,11)$ constitute the generalized null-plane gauge condition.

The next step is to calculate Dirac's Brackets which are defined by

$$
\begin{aligned}
\{A(x), B(y)\}^{*} \equiv & \{A(x), B(y)\}-\int d^{3} z d^{3} w\left\{A(x), \Theta_{a}(z)\right\}\left[\left(\mathscr{O}^{-1}\right)\right]^{a b}(z, w) \\
& \left\{\Theta_{b}(w), B(y)\right\}
\end{aligned}
$$

Here $\left\{\Theta_{a}(z)\right\}$ denote the set of ten second class constraints of the theory and $\left[\left(\mathscr{O}^{-1}\right)\right]^{a b}(z, w)$ represent the inverse of the matrix formed with this set of constraints. The explicit evaluation of the inverse involves the knowledge of the inverse of the operators $\left(\partial_{-}^{x}\right)^{-1},\left(\partial_{-}^{x}\right)^{-2}$, and $\left(\partial_{-}^{x}\right)^{-3}$. To achieve a unique solution it is necessary and sufficient to impose $\partial_{-}^{x} A_{\mu}=0, \partial_{-}^{x} \partial_{-}^{x} A_{\mu}=0$, and $\partial_{-}^{x} \partial_{-}^{x} \partial_{-}^{x} A_{\mu}=0$ on $x^{+} \rightarrow-\infty$ as the appropriate boundary conditions of the theory.

Thus, we have that the fundamental DB among the independent dynamical variables of the theory are:

$$
\begin{aligned}
\left\{A_{+}(x), \bar{A}_{k}(y)\right\}_{D} & =\frac{1}{32 a^{2}} \partial_{k}^{x} \delta^{2}\left(x^{\top}-y^{\top}\right) \partial_{-}^{x} \int d v^{-}\left|x^{-}-v^{-}\right|\left|v^{-}-y^{-}\right| \\
\left\{A_{+}(x), \pi^{-}(y)\right\}_{D} & =-\frac{1}{2} \varepsilon\left(x^{-}-y^{-}\right) \delta^{2}\left(x^{\top}-y^{\top}\right) \\
\left\{A_{k}(x), p^{l}(y)\right\}_{D} & =\delta_{k}^{l} \delta^{3}(x-y)
\end{aligned}
$$




$$
\begin{aligned}
\left\{\bar{A}_{k}(x), A_{l}(y)\right\}_{D}= & \frac{1}{16 a^{2}} \delta_{k}^{l}\left|x^{-}-y^{-}\right| \delta^{2}\left(x^{\top}-y^{\top}\right) \\
\left\{\bar{A}_{k}(x), \bar{A}_{l}(y)\right\}_{D}= & -\frac{\delta_{l}^{k}}{128 a^{4}}\left(x^{-}-y^{-}\right)^{2} \varepsilon\left(x^{-}-y^{-}\right)\left(1+4 a^{2} \nabla_{x}^{2}\right) \delta^{2}\left(x^{\top}-y^{\top}\right) \\
& +\frac{1}{16 a^{2}} \partial_{k}^{x} \partial_{l}^{x} \delta^{2}\left(x^{\top}-y^{\top}\right) 2 \partial_{-}^{x} \int d v^{-}\left|x^{-}-v^{-}\right|\left|v^{-}-y^{-}\right| \\
\left\{\bar{A}_{k}(x), p^{l}(y)\right\}_{D}= & -\frac{1}{16 a^{2}} \varepsilon\left(x^{-}-y^{-}\right)\left[-\delta_{k}^{l}+4 a^{2}\left(\partial_{l}^{x} \partial_{k}^{x}-\delta_{k}^{l} \nabla_{x}^{2}\right)\right] \delta^{2}\left(x^{\top}-y^{\top}\right) \\
\left\{\bar{A}_{k}(x), \pi^{-}(y)\right\}_{D}= & -\frac{1}{4} \varepsilon\left(x^{-}-y^{-}\right) \partial_{k}^{x} \delta^{2}\left(x^{\top}-y^{\top}\right)
\end{aligned}
$$

With these brackets we can deduce the fundamental ones that will lead, through the correspondence principle, to a consistent quantization of the field.

\section{FINAL REMARKS}

In the canonical analysis of the Podolsky's theory we found a set of three first-class constraints (7), followed by another set of four second-class ones (8) which are not present in the conventional instant-form dynamics [3]. The appearance of this kind of constraints is a common effect of the null-plane dynamics [7].

We eliminated the first class constraints (7) from our theory using the generalized nullplane gauge condition. By imposing the value of the field $A_{\mu}$ on the null-plane $x^{+}=$cte, and the considered boundary conditions: $\partial_{-} A_{\mu}=0,\left(\partial_{-}\right)^{2} A_{\mu}=0$, and $\left(\partial_{-}\right)^{3} A_{\mu}=0$ on $x^{+} \rightarrow-\infty$, on $x^{-}=$cte , we have fixed the hidden subset of the first-class constraints [9] and got a unique set of Dirac's brackets among the fundamental fields of the theory.

\section{ACKNOWLEDGMENTS}

M. C. Bertin was supported by CAPES. B. M. Pimentel was partially supported by CNPq. and G. R. Zambrano thanks the XI Hadron Physics organizing committee for partial financial support to attend this Meeting.

\section{REFERENCES}

1. B. Podolsky, P. Schwed, Rev. Mod. Phys. 20, 40 (1948). F. Bopp, Ann. Phys. 38, 345 (1940).

2. R. R. Cuzinatto, C. A. M. de Melo, P. J. Pompeia, Ann. Phys. 322, 1211 (2007).

3. C. A. P. Galvão, B. M. Pimentel, Can. J. Phys. 66, 460 (1988).

4. P. A. M. Dirac, Lectures on Quantum Mechanics, Yeshiva University, New York, (1964).

5. M. C. Bertin, B. M. Pimentel, G. E. R. Zambrano, The Canonical structure of Podolsky's generalized electrodynamics on the Null-Plane, arXiv: 0907.1078 [hep-th].

6. P. A. M. Dirac, Rev. Mod. Phys. 21, 392 (1949).

7. R. Casana, B. M. Pimentel, G. E. R. Zambrano, Int. J. Mod. Phys. E16, 2993 (2007).

8. R. A. Neville and F. Rohrlich, Pyis. Rev., D3, 1692 (1971).

9. R. Benguria, P. Cordero and C. Teitelboim, Nucl. Phys., B122, 61 (1976). 
Copyright of AIP Conference Proceedings is the property of American Institute of Physics and its content may not be copied or emailed to multiple sites or posted to a listserv without the copyright holder's express written permission. However, users may print, download, or email articles for individual use. 